\title{
Reversible Inactivation of the Cerebellar Interpositus Nucleus Completely Prevents Acquisition of the Classically Conditioned Eye-Blink Response
}

David J. Krupa ${ }^{1}$ and Richard F. Thompson

Neurosciences Program

University of Southern California

Los Angeles, California 90089-2520

\begin{abstract}
Numerous studies from several laboratories report that temporary inactivation of the cerebellar interpositus nucleus and regions of overlying cortex during eye-blink conditioning completely prevents acquisition of the conditioned eye-blink response (CR) without affecting the ability to learn the $C R$ in subsequent training without inactivation. Recently, these results have been challenged by the suggestion that learning was not completely blocked in these studies. Instead, it has been suggested that low levels of responses on test sessions might represent a retarded form of learning caused by drug effects on cerebellar cortex. The present study was designed to address this issue directly. Very low doses of muscimol were used to selectively inactivate the interpositus nucleus of rabbits during five conditioning sessions. Animals performed no significant levels of CRs during those sessions. Training was continued four more sessions without any inactivations to test whether any learning had occurred during the previous five sessions. Detailed analysis of responses during session six revealed that learning was completely blocked by the low doses of muscimol infused into the interpositus during the first five sessions. Animals subsequently acquired the CR normally.
\end{abstract}

${ }^{1}$ Corresponding author. Present address: Department of Neurobiology, Duke University Medical Center, Durham, North Carolina 27710.
These results confirm and extend the original findings that appropriate lesions (either temporary or permanent) of the interpositus nucleus completely prevent acquisition of the conditioned eye-blink response. Other issues regarding reversible inactivation studies are also discussed.

\section{Introduction}

Numerous lines of evidence, ranging in diversity from purely theoretical considerations (Marr 1969; Albus 1971) to human functional imaging studies (Molchan et al. 1994; Logan and Grafton 1995; Blaxton et al. 1996), have consistently implicated the cerebellum as being critically involved in a number of learning related tasks (for instance, see Lisberger 1988; Thatch et al. 1992; Thompson and Krupa 1994). Among the many tasks in which the cerebellum is critically involved, a number of studies from several laboratories have demonstrated that the cerebellum is essentially involved in acquisition and expression of classically conditioned discrete skeletal movements, in particular, the classically conditioned eye-blink response.

Briefly, the evidence supporting cerebellar involvement in eye-blink conditioning includes the following: (1) Appropriate lesions of a restricted region of lateral cerebellar hemisphere, including lesions limited to the anterior interpositus nucleus, completely and permanently abolish both acquisition and expression of conditioned eye-blink responses (CRs) without affecting performance of the reflexive, unconditioned response (UR) (Lincoln et al. 1982; McCormick and Thompson 1984a; Lavond et al. 1985; Yeo et al. 1985; Steinmetz et al. 1992). (2) Electrophysiological recordings of neuronal activity from within this region of cerebellum

LEARNING \& MEMORY 3:545-556 @ 1997 by Cold Spring Harbor Laboratory Press ISSN1072-0502/97 \$5.00

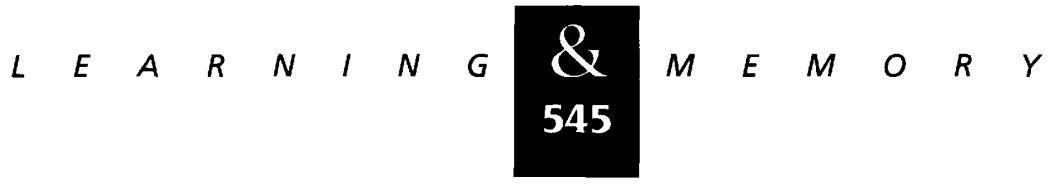


reveal populations of neurons that respond not only to conditioned and unconditioned stimuli but also during performance of CRs in a manner that precedes and predicts their occurrence, suggesting a causal role (McCormick and Thompson 1984b; Berthier and Moore 1986, 1990; Foy et al. 1992). (3) Electrical microstimulation of brain stem precerebellar nuclei or fibers immediately afferent to the cerebellum serves as an effective conditioned stimulus (CS) or unconditioned stimulus (US), depending on stimulus location. CRs elicited by these stimuli are completely abolished by appropriate, focal lesions of lateral cerebellum (Mauk et al. 1986; Steinmetz et al. 1986; Lavond et al. 1987). Collectively, these results demonstrate that the cerebellum is necessary for both acquisition and expression of the eye-blink CR.

Recently, several studies, each using reversible inactivation techniques, have provided very compelling evidence that the essential locus of memory formation and storage (i.e., the memory trace) for this type of learning is located within the restricted region of lateral cerebellum encompassing the interpositus nucleus and localized regions of overlying cerebellar cortex that project to the interpositus nucleus. Clark et al. (1992) initially reported that temporary, reversible inactivation (by local cooling) of this localized region of cerebellum during eye-blink conditioning in rabbits completely prevented acquisition of the eye-blink CR without affecting the ability to acquire the CR following removal of the cooling and without affecting the ability to perform the UR. This initial result was subsequently confirmed and extended by several studies, each using different techniques such as local microinjection into the anterior interpositus nucleus of muscimol, baclofen, or lidocaine to focally and reversibly inactivate this region of cerebellum (Krupa et al. 1993; Nordholm et al. 1993; Hardiman et al. 1996; Ramirez et al. 1997). The results of each of these studies were the same: Appropriate inactivation of the critical region of cerebellum completely prevented acquisition of the eye-blink CR with no effect on subsequent learning following removal of inactivation and with no effect on the ability to perform the UR. These results indicate that the memory trace for this type of learning must be localized either (1) within the region of cerebellum that was inactivated in these studies or (2) in some other structure(s) downstream from this region of cerebellum that receives cerebellar input that is essential for both acquisition and expression of the CR.
Subsequent studies have largely ruled out this second possibility. Reversibly inactivating the brain-stem motor nuclei and surrounding reticular formation responsible for performance of the CR has no effect at all on the ability to acquire the CR but does block CR expression, thus ruling out these structures as possible loci for the memory trace (Zhang and Lavond 1991; Krupa et al. 1996). Similarly, inactivation of the magnocellular red nucleus, a structure that receives direct input from the interpositus and is essentially involved in eyeblink conditioning, also prevents CR expression with no effect on CR acquisition (Clark and Lavond 1993; Krupa et al. 1993). Finally, inactivation of all of the output fibers of the interpositus nucleus during conditioning by either injection of tetrodotoxin in the superior cerebellar peduncle or lidocaine into the white matter ventral to the interpositus has the same effect: complete blockade of CR expression with no effect on CR acquisition (Nordholm et al. 1993; Krupa and Thompson 1995). Collectively, these results, along with the cerebellar reversible inactivation results (above), provide compelling data in support of a cerebellar memory trace: Inactivating this localized region of cerebellum (including the interpositus nucleus and overlying cortex) during conditioning completely prevents learning from occurring, whereas inactivating essential structures in the eyeblink circuit that are downstream from the cerebellum (including all of the output from this region of cerebellum) has no effect on the ability to learn the CR but does prevent $C R$ expression.

Recently, Bloedel and Bracha (1995) have suggested that our previous reversible inactivation studies (in which the interpositus nucleus and localized regions of overlying cortex were reversibly inactivated with either muscimol or lidocaine; Krupa et al. 1993; Nordholm et al. 1993) did not completely prevent learning from occurring but, instead, resulted in "...a low level of conditioned responses during the retention testing [which] reflects not an absence of learning but rather a 'retarded' process of learning caused by the drug effect on the cerebellar cortex"'(p. 12). Although the data from those previous studies directly and completely contradict this suggestion (see Discussion), the present study was designed to test this possibility in even more detail. Here, we used a very low dose of muscimol to selectively inactivate the interpositus nucleus during five sessions of eye-blink conditioning. Retention tests (which would reveal any signs of learning even on the first trial of test-

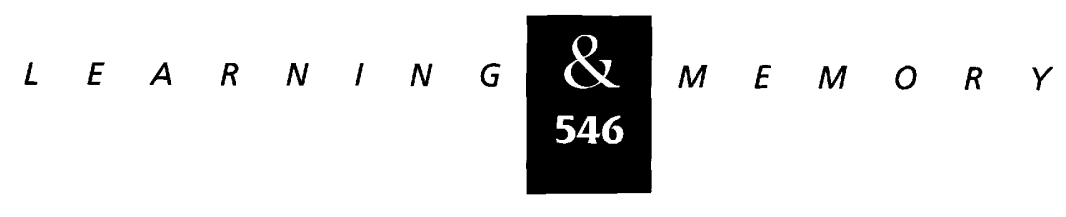


ing) following removal of this inactivation revealed no evidence at all of learning with no effect at all on the rate of subsequent learning in the absence of inactivation. The present results, therefore, confirm and extend the previous results of this and other laboratories; namely, appropriate lesions (either temporary or permanent) of a restricted region of lateral cerebellum, encompassing minimally the anterior interpositus nucleus, completely prevent acquisition of the classically conditioned eye-blink response and have no effect on performance of the reflex UR.

\section{Materials and Methods}

\section{SURGICAL PROCEDURES}

Under aseptic surgical procedures, $16 \mathrm{New}$ Zealand albino rabbits (Oryctolagus cuniculus, $\sim 2.5 \mathrm{~kg}$ at time of surgery) were each implanted with a chronic, stainless steel guide cannula (25 gauge, $0.65 \mathrm{~mm}$ o.d.) fitted with an internal stainless steel stylet (to ensure patency) that extended $1.5 \mathrm{~mm}$ beyond the base of the guide cannulae. Surgical procedures consisted of a midline incision through the scalp, retraction of the periostium, and a small craniotomy $(1.5 \mathrm{~mm}$ diam.) above the stereotaxic coordinates of the interpositus nucleus. The stylet tip was aimed at the dorsal aspects of the anterior region of the left cerebellar interpositus nucleus, stereotaxically positioned $0.7 \mathrm{~mm}$ anterior, $5.8 \mathrm{~mm}$ lateral, and $14.5 \mathrm{~mm}$ ventral to the $\lambda$ skull suture with $\lambda$ positioned $1.5 \mathrm{~mm}$ below bregma according to the stereotaxic atlas of McBride and Klemm (1968). The cannulae were anchored to the skull using dental acrylic and three stainless steel skull screws. A small receptacle for attaching a minitorque potentiometer and an airpuff nozzle during the behavioral training sessions was also cemented to the skull. A $1.0-\mathrm{mm}$ loop of 6-0 surgical suture (Ethilon) was placed in the apex of the left nictitating membrane (NM). Surgical anesthesia consisted of ketamine $(60 \mathrm{mg} /$ $\mathrm{kg}$ ), xylazine $(8 \mathrm{mg} / \mathrm{kg})$, and halothane (1\%-3\% in oxygen). All animals were treated in accordance with National Institutes of Health (NIH) guidelines. All rabbits received 7 days postoperative recovery.

\section{BEHAVIORAL TRAINING PROCEDURES}

Following postoperative recovery, rabbits received (on day 8 ) one session of habituation to restraint in a Plexiglas restrainer and the sound attenuating behavioral recording chamber for 1 hour. Rabbits were randomly assigned to one of two groups. One group ( $n=8$; termed the "Muscimol" group) received five daily tone-air-puff conditioning sessions during which the interpositus nucleus was reversibly inactivated with a microinjection of the $\gamma$-amino-butyric acid (GABA) agonist muscimol (see below). These rabbits then received 2 days of rest followed by four more daily conditioning sessions witbout any inactivation to test whether any learning had occurred during the previous five sessions with inactivation. Finally, these rabbits received a last conditioning session in which muscimol was again infused into the interpositus to test the effects of interpositus inactivation on retention of the CR and performance of the UR.

The second group ( $n=8$ ) of rabbits (termed the "Control" group) received an infusion of saline vehicle $(0.1 \mu \mathrm{l})$ into the interpositus nucleus before each of the first five control sessions. During each of these five control sessions, the rabbits were restrained and placed in the behavioral recording chamber but no stimuli were presented. Rabbits remained restrained in the chamber for the same length of time as the Muscimol group, $\sim 60$ min per session. Spontaneous eye-blink activity was recorded during these sessions by recording nictitating membrane movement at intervals identical to those in which stimuli were presented to the rabbits in the Muscimol group. Following these five sessions, rabbits in the Control group also received 2 days rest followed by four tone-air-puff conditioning sessions. As with the Muscimol group, no infusions were administered during these sessions. Finally, Control rabbits also received one last conditioning session in which muscimol ( 1 nmole) was infused into the interpositus to test the effects on retention of the CR and performance of the UR.

Each tone-air-puff conditioning session consisted of 100 trials divided into 10 blocks of 10 trials. Each block of 10 trials consisted of 1-tone alone trial followed by 4 paired tone-air-puff trials followed by 1 air-puff alone trial followed by 4 more paired tone-air-puff trials. Intertrial interval varied randomly between $20 \mathrm{sec}$ and $40 \mathrm{sec}$ [mean $(M)=30 \mathrm{sec}$. Paired tone-air-puff trials consisted of a tone CS (350 msec, $1 \mathrm{kHz}, 85 \mathrm{~dB})$ paired with a coterminating corneal air-puff US (100 msec, 2.1 $\mathrm{N} / \mathrm{cm}^{2}$ pressure at the source). The tone was presented through a small loudspeaker placed $30 \mathrm{~cm}$

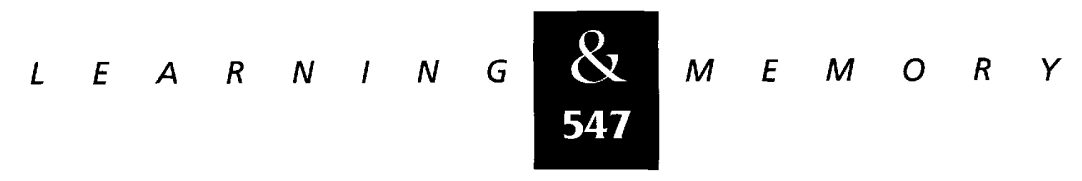


in front of the rabbit. The air puff was delivered through a 3-mm i.d. tube positioned $1 \mathrm{~cm}$ from the center of the rabbit's cornea. Behavioral responses were measured with a minitorque potentiometer attached to the suture loop in the rabbits' left NM. CRs were defined as any $0.5-\mathrm{mm}$ or greater extension of the NM occurring anytime between 35 msec and $250 \mathrm{msec}$ (US onset) after CS onset. On CS alone trials, a CR was counted as any response $\geqslant 0.5 \mathrm{~mm}$ occurring anytime between $35 \mathrm{msec}$ and $750 \mathrm{msec}$ after CS onset. On both paired and tone alone trials, any response occurring between 0 msec and $35 \mathrm{msec}$ after CS onset was considered as an $\alpha$ response or a spontaneous response and was not counted as a CR. URs were defined as any movement of the NM within $500 \mathrm{msec}$ following US onset (the minimum resolvable movement was $100 \mu \mathrm{m})$.

\section{MUSCIMOL INFUSIONS}

One hour prior to each of the first five training sessions, rabbits in the Muscimol group received an infusion of muscimol $(1.0 \mathrm{nmole}$ in $0.1 \mu \mathrm{l}$ of isotonic saline vehicle; Sigma) into the left interpositus nucleus. This low dose of muscimol was chosen (based on previous work) to inactivate the interpositus nucleus without diffusing throughout the overlying cerebellar cortex and inactivating that region of cerebellum. Rabbits in the Control group were infused with $0.1 \mu \mathrm{l}$ of saline vehicle 1 hr prior to each of the first five sessions. Infusion procedures for all rabbits involved removal of the internal stylet from the guide cannula, insertion of a stainless steel injector cannula (31 ga, $0.21 \mathrm{~mm}$ o.d.) that extended $1.5 \mathrm{~mm}$ below the base of the outer guide cannula, infusion of the drug at 0.3 $\mu \mathrm{l} / \mathrm{min}$, removal of the injector cannula $3 \mathrm{~min}$ after cessation of infusion, and, finally, reinsertion of the internal stylet.

\section{HISTOLOGY}

To determine the extent of diffusion of the 1.0-nmole dose of muscimol in cerebellum, ${ }^{3} \mathrm{H}$-labeled muscimol was infused into the interpositus nucleus of six of the eight rabbits in the Muscimol group. Each rabbit was infused with 1.0 nmole (in $0.1 \mu \mathrm{l}$ of saline) of ${ }^{3} \mathrm{H}$-labeled muscimol on the day after the tenth training session. In each infusion, tracer doses of ${ }^{3} \mathrm{H}$-labeled muscimol $(2 \mu \mathrm{Ci} / \mu \mathrm{g}$; NEN) were mixed with the unlabeled muscimol. One hour after infusion, each rabbit received 50 training trials (40 paired, $5 \mathrm{CS}$ alone, and $5 \mathrm{US}$ alone) to ensure that the radiolabeled muscimol effectively abolished the CR acquired previously (as did the previous, nonradioactive infusions). Immediately after the fiftieth trial, each rabbit was lightly anesthetized with halothane and decapitated. Their brains were removed rapidly and then frozen in liquid isopentane on dry ice. The frozen brains were sectioned on a cryostat $(20 \mu \mathrm{m})$. The frozen sections, along with ${ }^{3} \mathrm{H}$ standards (Amersham), were apposed to X-ray film (Amersham Hyperfilm) for 21 days. The exposed films were developed, and the brain sections were fixed in formalin fumes and stained with cresyl violet. The extent of muscimol diffusion throughout the interpositus/dentate nuclei was then determined.

Following all training sessions, all rabbits in the Control group (and the two rabbits in the Muscimol group not infused with ${ }^{3} \mathrm{H}$-labeled muscimol) were injected intravenously with a lethal dose of sodium pentobarbital, then perfused through the aorta with $0.9 \%$ saline followed by a $10 \%$ formalin solution. The position of the stylet tips was marked by passing $80 \mu \mathrm{A}$ (for $8 \mathrm{sec}$ ) of anodal current through a stainless steel lesioning electrode that had been lowered through the guide cannula to the exact depth of the stylet tip. This position is the same as that of the inner injection cannula tip when it was fully lowered during saline and muscimol infusions. The brains were then removed, embedded in an albumen gel, and stored in 10\% formalin until they were sectioned $(80 \mu \mathrm{m})$ on a freezing microtome. The sections were stained with cresyl violet and Prussian blue, and the location of the stylet tip was determined.

\section{DATA ANALYSIS}

The mean percentage of CRs was calculated for the paired trials for each block and each session. Data were analyzed with mixed analyses of variance with Group as the between subjects factor and Session or Block as the repeated measure. Where appropriate, post hoc Newman-Keuls tests were used to further analyze significant main effects or interactions. A significance level of 0.05 was used for all statistical tests.

\section{Results}

Cannula placements of two of the rabbits in the Muscimol group were located outside the in-

\begin{tabular}{lllllllllllllllll}
\hline & $E$ & $A$ & $R$ & $N$ & $I$ & $N$ & $G$ & $\underset{548}{\mathbf{Z}}$ & $M$ & $E$ & $M$ & $O$ & $R$ & $Y$
\end{tabular}


terpositus nucleus in the lateral ansiform lobule. Both of these rabbits showed significant levels of learning during the first five conditioning sessions in which muscimol was infused, each exceeding at least $\mathbf{4 1 \%}$ CRs during at least one session and each reaching a learning criterion of eight $C R s$ in nine consecutive trials. In one rabbit, infusion of muscimol on session 10 had no effect at all on CR performance relative to performance during session 9 in which no infusions were administered ( $94 \%$ on session 9 vs. $100 \%$ on session 10 ). Examination of the spread of ${ }^{3} \mathrm{H}$-labeled muscimol in this rabbit revealed labeling predominantly located in ansiform cortex with very low concentrations of muscimol in the dorsal aspects of the dentate nucleus. No labeling was detected in the interpositus nucleus. CRs of the other rabbit on session 10 (59\%) were lower than on session 9 (96\%) but were not completely abolished. Analysis of the spread of ${ }^{3} \mathrm{H}$-labeled muscimol in this rabbit revealed the highest concentration of muscimol in ansiform cortex as well as a very low concentration of muscimol in the most dorsal-lateral aspects of the interpositus nucleus and dorsal aspects of the dentate. Most of the interpositus nucleus, however, was not labeled. On the basis of these criteria, it was concluded that the muscimol infusions during sessions 1-5 and 10 were not effectively inactivating the entire extent of the interpositus nucleus in these rabbits. These animals, therefore, were not included in further analyses. Similarly, the cannula placement of one rabbit in the control group was outside the interpositus. Infusion of muscimol on session 10 had no effect on CR performance (relative to session 9). This rabbit was also excluded from further analysis. One rabbit in the Control group dislodged its headstage prior to completion of conditioning and was, therefore, excluded from further study.

Infusions of muscimol into the interpositus nucleus of the remaining six rabbits in the Muscimol group before each of the first five sessions completely prevented any expression of eye-blink CRs during those sessions (Fig. 1A). Responses during those sessions did not differ significantly from the spontaneous levels recorded in the Control group that was restrained and placed in the behavioral recording chamber during sessions $1-5$ but presented no stimuli. An ANOVA with factors Group and Session yielded no significant main effects of Group, Session, or their interaction.

On session 6 , the first session without any inactivation, rabbits in the Muscimol group showed

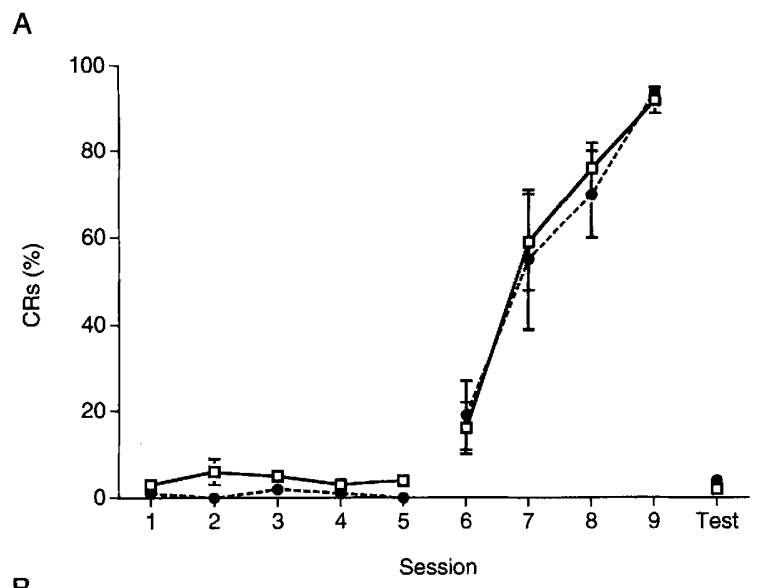

B

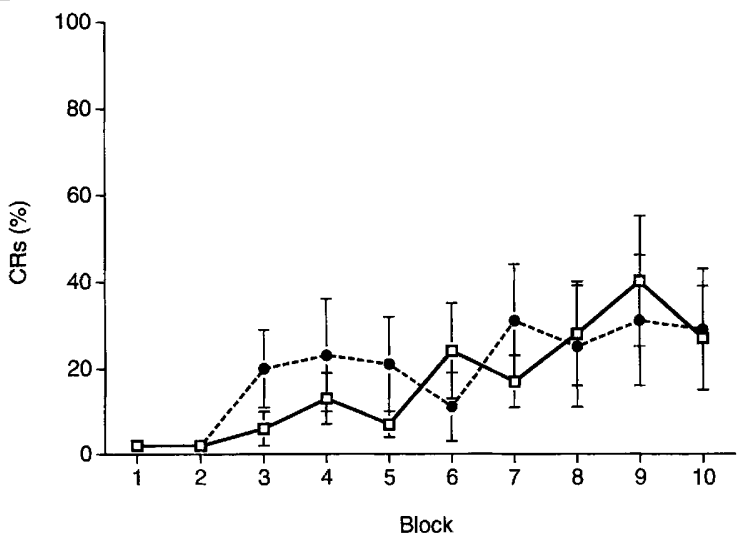

Figure 1: (A) Mean ( \pm S.E.) percentage CRs for Muscimol and Control Groups for each training session. On sessions 1-5, rabbits received microinjections of $1.0-$ nmole muscimol [Muscimol group ( $\square$ ]) or saline vehicle [Control group (-)] into the interpositus nucleus. The Muscimol group was presented with paired tone-airpuff training during inactivation, whereas the Control group received no stimuli. On sessions 6-9, no infusions were administered and both groups received paired training. On the Test session, both groups received an injection of muscimol ( 1 nmole) to assess its effects on retention of the CR. There were no differences between groups during sessions 6-9. Inactivation of the interpositus nucleus with this low dose of muscimol completely blocked acquisition of the eye-blink CR without affecting the ability to learn the $C R$ during sessions 6-9 during which no infusions were administered. (B) Mean ( \pm S.E.) percentage CRs for Muscimol and Control groups as a function of training block on session 6 (first session without infusion). Each block consisted of eight paired toneair-puff trials. Again, there were no differences between groups. Responses of the Muscimol group at the start of training were the same as Controls. Both Muscimol and Control rabbits also showed the same rate of acquisition during the session. There was no evidence at all that Muscimol animals had learned the CR during sessions $1-5$.

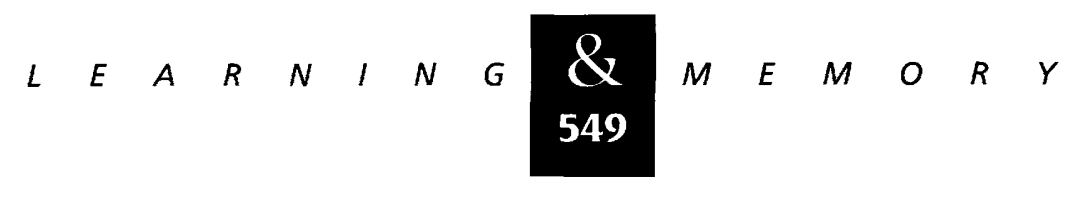


no signs of any learning from the previous five conditioning sessions (in which the interpositus was inactivated) and subsequently learned the CR as if naive. Their performance on session 6 and subsequent rate of acquisition on sessions 7-9 was indistinguishable from control rabbits that had been infused with saline on sessions 1-5 but presented with no stimuli on those sessions. A $t$-test on trials to criterion (eight CRs in nine consecutive trials) for the two groups revealed no significant difference in learning rate $[t(10)=0.29, P>0.05$; mean \pm S.D. for Muscimol group $=153 \pm 74$ trials, Control group $=140 \pm 80$ ). Again, an ANOVA with factors Group and Session revealed no significant effect of Group or interaction $(F$ values $<1$ ), but in this case, a strong effect of Session was found $[F(3,30)=52.4, P<0.0001]$, indicating that significant acquisition had occurred over the 4 days of training. Infusion of muscimol into the interpositus of both Muscimol rabbits and Control rabbits on session 10 (Test) completely abolished the CR learned during sessions 6-9 (see Fig. 1A).

To ensure that the low level of CRs performed by the Muscimol group on session $6(16 \%)$ did not represent a "retarded" process of learning (as suggested by Bloedel and Bracha 1995; see Introduction) but, instead, simply reflected the normal CR acquisition that would be expected of naive animals during the course of a single training session, we compared the performance of the Muscimol group with the Control group on a block-by-block and a trial-by-trial basis. If any learning had occurred during sessions $1-5$, it would be manifest, on session 6 , in the form of a higher rate of responding by the Muscimol group during the initial trials of session 6 and/or a higher rate of acquisition over the course of that (and/or subsequent) sessions. The results clearly demonstrate that neither occurred. A block-by-block (each training block consisted of eight paired tone-air-puff trials) analysis of the mean percentage of CRs for each group revealed no differences at all between groups (Fig. 1B). A Group $\times$ Block ANOVA yielded no significant effect of Group or interaction $[F(1,10)<1$ and $F(9,90)=1]$, but a significant effect of Block was found $[F(9,90)=4.98$, $P<0.0001]$, indicating that significant acquisition occurred over blocks on the first day of training without inactivation. Furthermore, trial by trial analysis of responses on tone alone test trials during session 6 also revealed no differences at all between groups and no significant interaction of Group and Trial (both $F$ values $<1$ ). Again, signifi- cant acquisition was seen across the 10 tone alone trials of session $6[F(9,90)=2.63, P<0.01]$. This tone alone test trials measure is particularly sensitive because it includes any responses for a period up to $750 \mathrm{msec}$ after CS onset. Therefore, if rabbits in the Muscimol group were performing any long latency CRs, these would be detected during the tone alone test trials. However, no difference was seen between groups on this or any other measure of acquisition. In summary, inactivation of the interpositus nucleus with a very low dose of muscimol completely prevented learning from occurring without any effects at all on the ability to acquire the $C R$ in subsequent training without inactivation.

Consistent with previous results, infusions of muscimol into the interpositus nucleus had no effect on the performance of the air puff-evoked UR (Fig. 2). There was no significant difference between UR amplitudes of the Muscimol group (recorded on air-puff alone test trials) on session 5 during which muscimol infusions inactivated the interpositus and session 6 in which no infusions were administered $[t(5)=-0.04, P>0.05]$. (Because Control animals were not presented with

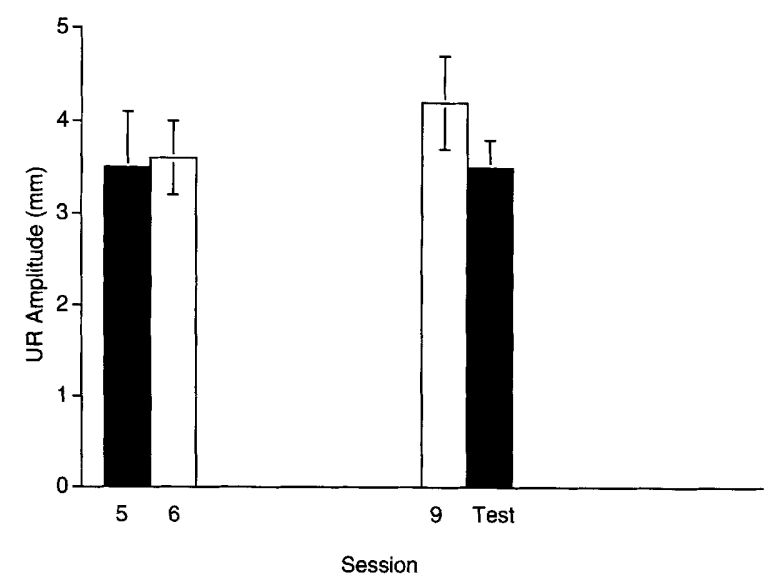

Figure 2: Mean ( \pm S.E.) $\cup R$ amplitudes measured on airpuff alone trials for the Muscimol group on session 5 (last session with inactivation) and session 6 (first training session without inactivation). Mean UR amplitudes for session 9 (last training session without inactivation) and Test session (with inactivation) are collapsed across group, because both groups were treated identically in this phase of the experiment and there were no significant differences between groups. Inactivation of the interpositus nucleus with 1 nmole of muscimol had no effect on the ability to perform the reflexive air puffevoked UR. Solid bars indicate sessions with muscimol infusion; open bars indicate sessions without muscimol infusion.

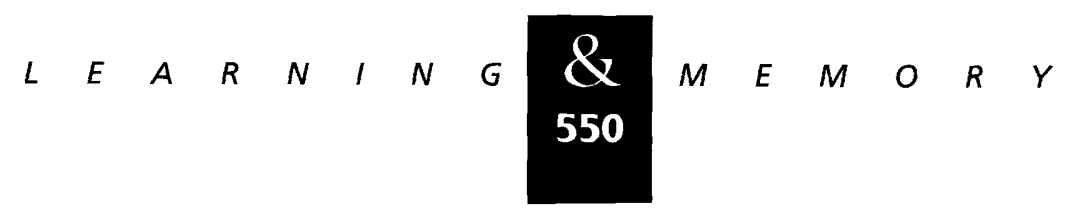


any stimuli during sessions 1-5, comparisons of UR amplitudes between groups cannot be made for these sessions.) There were also no significant differences between UR amplitudes recorded on session 9 (no infusion) and session 10 in which muscimol was infused into the interpositus nucleus of both groups to test retention [no significant effect of Group, $F(1,10)=2.2$; Session, $F(1,10)=1.7$; or their interaction, $F(1,10)=2.3$; all $P$ values $>0.05]$.

Histological reconstructions of all cannula placements are shown in Figure 3A. Effective placements are located within or just adjacent to
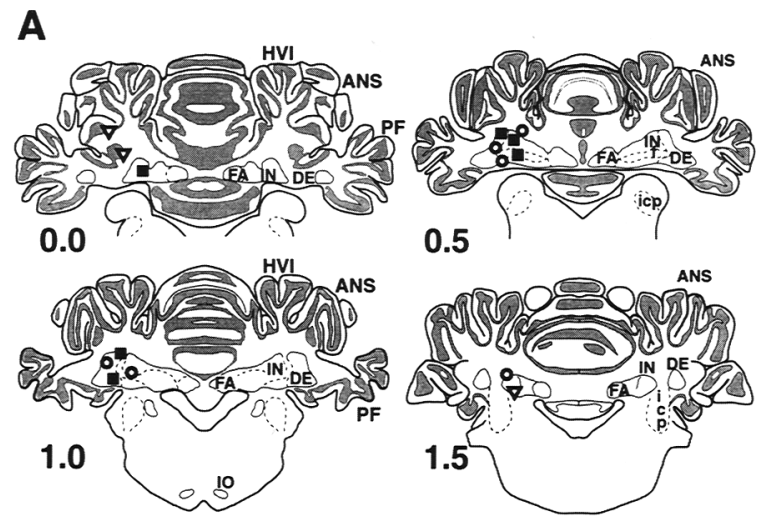

B
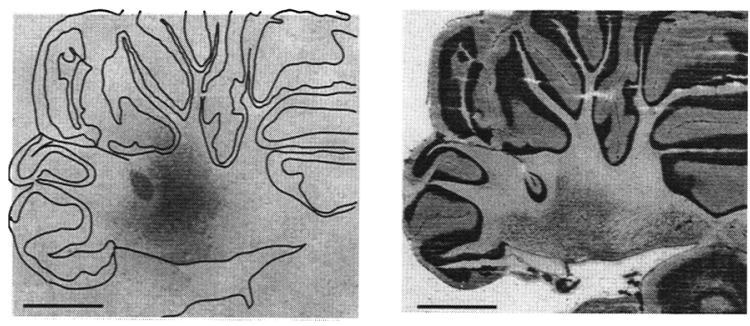

Figure 3: (A) Cannula locations for each of the rabbits. (西) Effective muscimol infusion sites; $(\bigcirc)$ controls; $(\nabla)$ ineffective placements. Abbreviations: (ANS) ansiform cortex; (DE) dentate nucleus; (FA) fastigial nucleus; (HVI) hemispheric lobule $\mathrm{Vl}$; (icp) inferior cerebellar peduncle; (IN) interpositus nucleus; (IO) inferior olive; (PF) paraflocculus. Numerals at bottom of standard sections represent distance $(\mathrm{mm})$ rostral from the $\lambda$ skull suture. (B) Autoradiograph (left) showing the largest extent of ${ }^{3} \mathrm{H}$-labeled muscimol diffusion. Superimposed on the autoradiograph is an outline drawing of the Nissl-stained section from which it was exposed (right). Diffusion of muscimol is restricted to the interpositus/dentate nuclei with very low levels of muscimol diffusion into the most ventral aspects of overlying ansiform cortex. There is no evidence at all of diffusion outside of the cerebellum. Calibration bar, $3.0 \mathrm{~mm}$. the anterior interpositus nucleus. In two rabbits (infused with muscimol during sessions 1-5), ineffective placements were located in ansiform cortex, dorsal and lateral to the interpositus nucleus. Infusion of muscimol into the more dorsal of these locations had no effect at all on acquisition or retention of the eye-blink CR. Muscimol infusion into the other location did not prevent acquisition or expression of the CR. However, muscimol infusion into this region did partially affect CR performance on session 10 (above). In one rabbit (infused with saline during sessions 1-5), the cannula was located just rostral and ventral to the anterior interpositus. Infusion of muscimol (on session 10) in this rabbit had no effect on performance of the CR.

To determine the maximal extent of muscimol diffusion, ${ }^{3} \mathrm{H}$-labeled muscimol was infused into the interpositus nuclei of four rabbits with effective cannula placements. ${ }^{3} \mathrm{H}$-labeled muscimol was also infused into the two Muscimol rabbits with ineffective placements (see above). Figure 3B (left) shows the autoradiograph of the largest extent of diffusion of ${ }^{3} \mathrm{H}$-labeled muscimol. Muscimol diffused throughout the anterior interpositus nucleus as well as regions of the dentate nucleus. In this particular rabbit, there was a very low concentration of muscimol in the most ventral aspects of ansiform cortex (also see Fig. 4, top row). There was no diffusion outside of the cerebellum. The maximal extent of muscimol diffusion for each of the four rabbits with effective cannula placements that were infused with ${ }^{3} \mathrm{H}$-labeled muscimol is shown in Figure 4 . In each case, diffusion of ${ }^{3} \mathrm{H}$ labeled muscimol was restricted almost entirely to the interpositus/dentate nuclei. There was no evidence at all of muscimol diffusion outside of the cerebellum. These very localized patterns of diffusion were further confirmed by the ineffective placements. Infusion of muscimol through cannulae located just outside the interpositus had no effect on CR acquisition or expression.

\section{Discussion}

The results of the present study are clear: Inactivation of a very localized region of cerebellum, including dorsal aspects of the anterior interpositus nucleus, with a very low dose of muscimol completely prevented acquisition of the conditioned eye-blink response. Subsequent learning in the absence of muscimol was completely unaffected, ruling out the possibility of any lingering

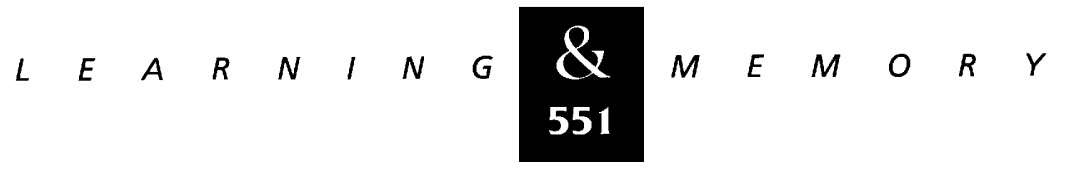



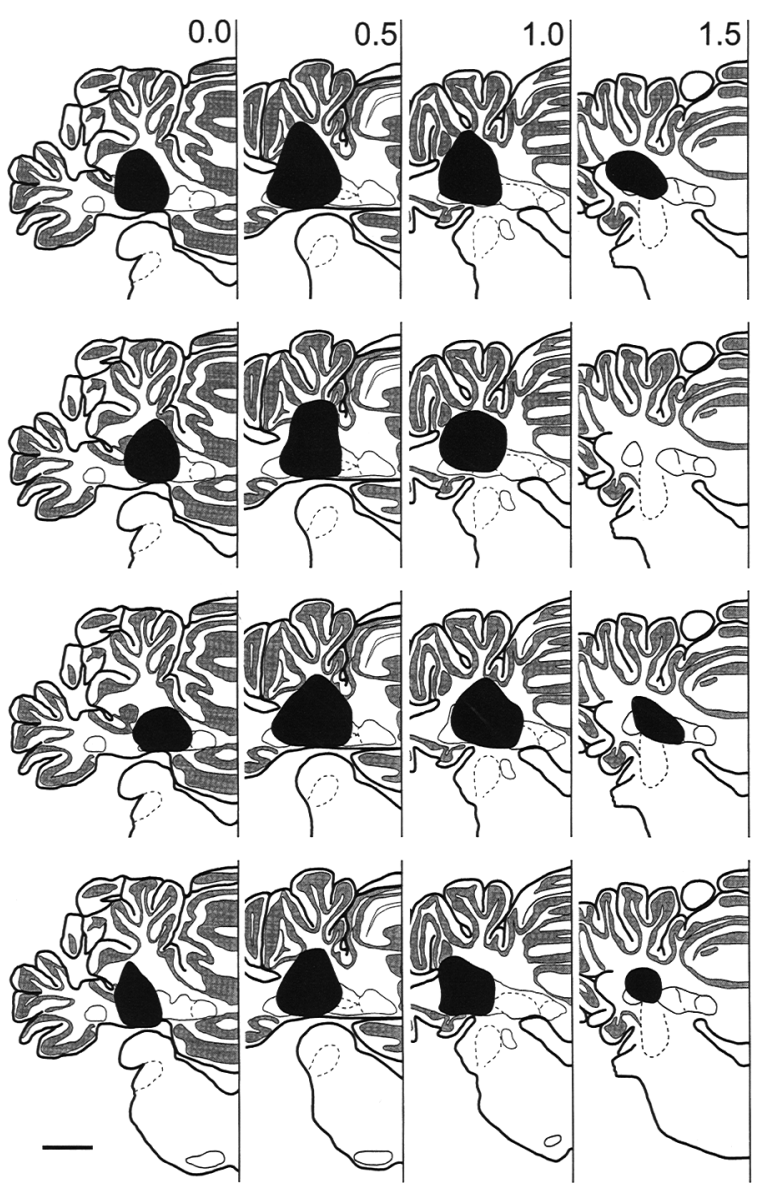

Figure 4: Maximal extent of ${ }^{3} \mathrm{H}$-labeled muscimol diffusion (shown on standard sections) in four rabbits with effective cannula placements. Muscimol diffused throughout the interpositus nucleus and regions of dentate nucleus. In some rabbits, very low concentrations of muscimol diffused into the most ventral aspects of ansiform cortex. There was no diffusion of muscimol outside of the cerebellum.

effects of muscimol. A block-by-block comparison of percent CRs over the first conditioning session without inactivation revealed no differences at all between the Muscimol and Control groups. There were also no differences on subsequent conditioning sessions. Examination of percent CRs on tone alone test trials during session 6 also revealed no differences between groups. This test is particularly sensitive because it would reveal any long latency CRs if they had developed. Muscimol inactivations also had no effect on the ability to perform the air puff-evoked UR. Quantitative autoradiography confirmed that the spread of muscimol was localized to the dentate/interpositus nuclei and, in some rabbits, the most ventral aspects of lateral cerebellar cortex. There was no spread of muscimol outside the cerebellum.

These results confirm and extend the results of previous studies from several laboratories in which reversible inactivation techniques were used to temporarily block cerebellar activity during eyeblink conditioning. Each of these studies used entirely different methods of inactivation: local cooling (Clark et al. 1992), lidocaine infusion (Nordholm et al. 1993), baclofen microinjection (Ramirez et al. 1997), and muscimol microinjection with air-puff US (Krupa et al. 1993) or muscimol microinjection with periorbital shock US (Hardiman et al. 1996). Despite these different techniques, the results of each study were the same: Cerebellar inactivation prevented acquisition of the eye-blink CR with no effect on the ability to learn the CR in subsequent training without inactivation.

The present data directly contradict the recent challenges by Bloedel and Bracha (1995) regarding the results of our (and other) previous studies. Those authors contend that we used "unusually high amounts of muscimol", thereby creating the possibility that the drug diffused to and inhibited neurons at extracerebellar sites. Furthermore, they suggest that because we inactivated regions of cerebellar cortex, "it is possible that a low level of conditioned responses during the retention testing reflects not an absence of learning but rather a 'retarded' process of learning caused by the drug effect on the cerebellar cortex." The authors, however, offer no evidence at all to support these claims. As described below, the results of the present study decisively rule out these arguments.

In our previous study (Krupa et al. 1993), the intent was to test whether eye-blink conditioning could occur while both the interpositus nucleus as well as regions of overlying cerebellar cortex were inactivated. To inactivate this region of cerebellum, we used a muscimol dose of 14 nmoles in $\mathbf{1 . 0}$ $\mu l$. Although Bloedel and Bracha (1995) characterize this dose as "unusually high" and suggest that muscimol might have diffused out of the cerebellum and inactivated extracerebellar sites, all of the available data (including data from their own laboratory) argue against this possibility.

For instance, prior to our study, a number of other laboratories had previously used doses of muscimol similar to or greater than ours to selectively inactivate localized regions within the brain including similar regions of cerebellum (Hikosaka and Wurtz 1985a,b; Martin and Ghez 1988; Van

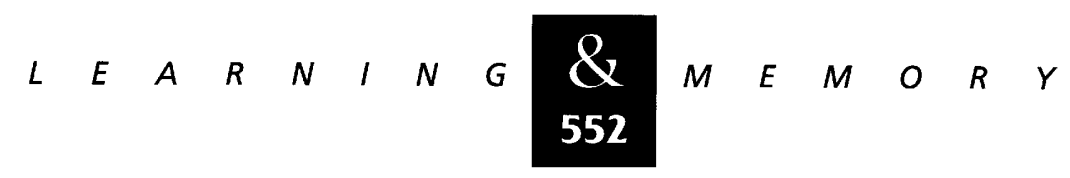


Neerven et al. 1989; Keating and Thach 1991; Mink and Thach 1991), contradicting the suggestion that our dose was unusually high. Martin (1991), using a combination of both ${ }^{3} \mathrm{H}$-labeled muscimol autoradiography and $\mathrm{L}-\left[{ }^{14} \mathrm{C}\right]$ glucose uptake reported that the maximal radial spread of a similar dose of muscimol in cerebral cortex was $\sim 2.5 \mathrm{~mm}$, a result similar to the spread of muscimol we reported (Krupa et al. 1993, p. 990; Fig. 2). Furthermore, Martin (1991) demonstrated that the radial spread of muscimol is very stable for at least $2 \mathrm{hr}$ after infusion, arguing against the possibility of muscimol spreading outside the cerebellum during the time course of our experiments.

Consistent with these results, our own autoradiographic analysis of the spread of ${ }^{3} \mathrm{H}$-labeled muscimol found no evidence at all of diffusion of muscimol outside of the cerebellum. Previous and subsequent work in our laboratory, combining electrophysiological recordings, behavioral measures, and quantitative autoradiography confirmed that infusion of 14 nmoles of muscimol into the interpositus nucleus inactivated this structure as well as regions of cerebellar cortex overlying this nucleus without diffusing outside the cerebellum (Krupa et al. 1992; Krupa 1993).

Finally, evidence from their own laboratory argues against the suggestion by Bloedel and Bracha that our dose of muscimol was unusually high. In their recent study, Bracha et al. (1994) used a dose of 3.5 nmoles to inactivate the interpositus nucleus. The authors estimated the effective radial spread of drug to be $\sim 1.6 \mathrm{~mm}$. In our study, the volume of cerebellum inactivated included both the interpositus nucleus as well as regions of overlying cerebellar cortex, a volume estimated to be 3.7 times the volume inactivated by Bracha et al. As such, the required dose of muscimol to inactivate this greater volume would be correspondingly larger. Therefore, the dose of 3.5 nmoles used by Bracha et al. to inactivate just the interpositus nucleus corresponds very well with our proportionally larger dose of 14 nmoles used to inactivate both the interpositus as well as overlying cortex. Thus, although Bloedel and Bracha characterize our dose of 14 nmoles as "unusually high", their own data demonstrate that it was not. In short, all of the available evidence, including the data of Bracha et al., indicates that the dose of muscimol (14 nmoles) used in our original study was precisely the dose necessary to inactivate the region of cerebellum intended. More importantly, there is simply no evidence whatsoever that muscimol dif- fused out of the cerebellum and inactivated extracerebellar sites.

In light of these results, the data from the present study conclusively rule out the possibility that inadvertent and undetected inactivation of extracerebellar sites by muscimol might be the reason for the complete prevention of eye-blink conditioning during cerebellar inactivation. Here, we used a very low dose of muscimol (1.0 nmole) to selectively inactivate the interpositus nucleus. In addition to using a very low dose, the infusion volume was also reduced to $0.1 \mu$, one-tenth the volume typically used in previous studies. As pointed out by Bracha et al. (1994), muscimol spread appears to be mainly attributable to replacement of extracerebellar fluid induced by injection pressure. Results of our autoradiographs are entirely consistent with this point. As such, the much lower volume of infusate used in the present study, coupled with the lower dose of muscimol, would result in a very restricted sphere of diffusion. This was confirmed by our autoradiography that demonstrated that the radial spread of muscimol was $\sim 1.5 \mathrm{~mm}$. In none of the autoradiographs was there any evidence at all of muscimol diffusion into any extracerebellar structures. In summary, these data argue decisively against the suggestion by Bloedel and Bracha (1995) that the complete and total prevention of eye-blink conditioning following appropriate cerebellar inactivation might be the result of muscimol diffusing to (and inactivating) extracerebellar sites during the course of the eye-blink conditioning sessions. There is absolutely no evidence of extracerebellar actions in any of the studies using reversible inactivation of the interpositus nucleus during training (Clark et al. 1992; Krupa et al. 1993; Nordholm et al. 1993; Hardiman et al. 1996; Ramirez et al. 1997). Finally, we note that Bracha et al. (1994) did not infuse muscimol during acquisition and is therefore irrelevant to the present issue of the role of the cerebellum in learning the CR.

The further suggestion by Bloedel and Bracha (1995) that the low level of CRs performed on the first session without inactivation might somehow represent a retarded form of learning is simply not supported by the data. In our previous study, the animals that had been infused with muscimol during the first six conditioning sessions performed an average of $19 \%$ CRs on session 7 , the first conditioning session without inactivation. However, these CRs merely represented the normal rate of responses that would be expected by naive animals over the first session of conditioning. This

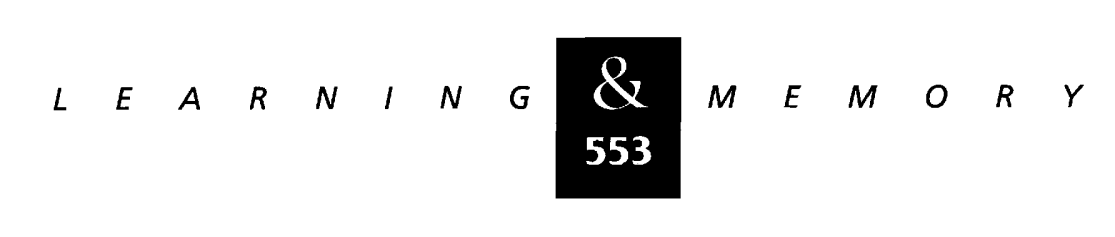


was confirmed by a block-by-block and trial-by-trial comparison of the performance of the muscimol group with control animals during that first session without inactivation. This comparison revealed no differences at all between groups.

The present study reinforces this result. On session 6, the first session without inactivation, the Muscimol group performed 16\% CRs. However, examination of those CRs clearly demonstrates that those responses represented a normal rate of acquisition expected during the first conditioning session. If any learning had occurred during sessions $1-5$, this would be expressed on sessions 6-9 in the form of either performance of a higher number of CRs early in session 6 and/or a faster rate of acquisition on session 6 and subsequent sessions. However, the data confirm that this did not occur. There were no differences at all between the Muscimol and Control groups in either the number of CRs performed early in training (including the very first trials) or in the rate of acquisition during session 6 and subsequent sessions. Comparison of performance on tone alone test trials revealed no differences between groups, ruling out the possibility that the Muscimol rabbits were performing long latency responses that would not be detected on paired tone-air-puff trials. The very low dose of muscimol used in this study was such that inactivation was limited to the interpositus nucleus. Muscimol did not inactivate overlying regions of cerebellar cortex shown previously to be involved in eyeblink conditioning. In summary, the present results decisively rule out the suggestions by Bloedel and Bracha (above) and confirm all of the previous results of this and other laboratories: Temporary inactivation of a restricted region of cerebellum, minimally encompassing the anterior interpositus nucleus, completely prevents acquisition of the eyeblink CR, with no effect on the UR and with no effect at all on the ability to learn the CR in subsequent training without inactivation.

Although the muscimol dose used here did not inactivate significant regions of cerebellar cortex, the results of the present study do not rule out a possible role for cerebellar cortex in acquisition or expression of the eyeblink CR. Several lines of evidence support the hypothesis that, during eyeblink conditioning, plasticity occurs in both the cerebellar cortex as well as the interpositus nucleus. For instance, permanent lesions of cerebellar cortex (which spare the interpositus nucleus) have been shown to significantly impair (and in some cases, completely prevent) acquisi- tion and expression of the CR. However, the precise functional roles of cerebellar cortex and the interpositus nucleus in eye-blink conditioning remain largely unknown. Further study will be required to elucidate the role of each of these structures in the process of eye-blink conditioning.

\section{MUSCIMOL-INDUCED DEPRESSION IN THE INTERPOSITUS NUCLEUS?}

Recently, Perrett and Mauk (1995) suggested that inactivation of the interpositus nucleus with muscimol during eye-blink conditioning did not block learning per se but instead resulted in a depression of mossy fiber synaptic efficacy within this nucleus that apparently masked plasticity that had occurred in cerebellar cortex during training. However, these authors offer no evidence to support this idea. Numerous lines of evidence rule out this possibility.

First, in our previous study, both the interpositus nucleus as well as cerebellar cortex were inactivated with muscimol during conditioning. In the present study, using a much lower dose of muscimol, only the interpositus nucleus was inactivated. However, percent CRs as well as the rate of CR acquisition on the first session without muscimol inactivation were identical for both groups. These measures were also identical to controls infused with saline. If muscimol infusions were somehow causing a synaptic depression within the interpositus, it would seem likely that there would be differences in CR performance by these different groups of rabbits, but there were none.

Second, other studies have used local cooling (Clark et al. 1992) and lidocaine (Nordholm et al. 1993) to inactivate the interpositus nucleus during eye-blink conditioning. These methods of inactivation would abolish both pre- as well as postsynaptic activity within the interpositus, which would prevent any form of synaptic plasticity from occurring. Use of these methods of inactivation also completely prevented eye-blink conditioning from occurring (above). The performance of animals on the first session without inactivation in these studies, however, was the same as performance of rabbits infused with muscimol. If muscimol infusions into the interpositus had resulted in a long-term depression, the rates of acquisition in each of these studies should be different, but they are not.

Third, Yeo and colleagues (Hardiman et al. 1996; Ramnani and Yeo 1996) recently tested the

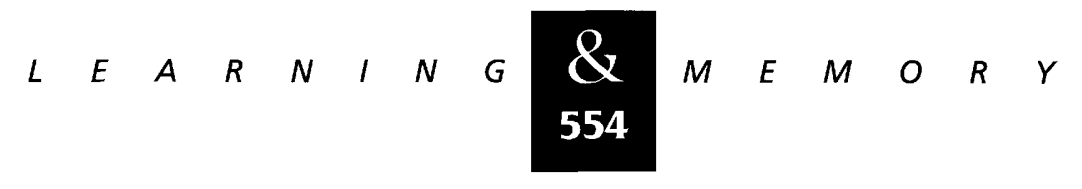


possibility of muscimol-induced depression directly. They first trained rabbits to perform the CR. They then infused muscimol into the cerebellum and presented the animals with tone alone extinction training. They then tested the animals without muscimol to determine whether any extinction had occurred. They found that the rabbits immediately performed CRs at preinfusion rates: No decrement in responding had occurred while the interpositus was inactivated with muscimol. This result alone would appear to rule out the possibility of a synaptic depression in the interpositus during these studies.

In summary, the present study and all other studies in which a localized region of the cerebellum including the anterior interpositus nucleus is inactivated during initial training of the conditioned eye-blink response agree that no learning occurs. All evidence to date strongly supports the hypothesis that the cerebellum is the site of memory storage for this form of learning, and there is no convincing evidence to the contrary (see Yeo 1991; Lavond et al. 1993; Thompson and Krupa 1994; Thompson and Kim 1996).

\section{Acknowledgments}

We thank Christine G. Logan for critical comments and Anne C. Krupa for invaluable assistance during preparation of this manuscript. This research was supported by National Science Foundation grant IBN-9215069, Office of Naval Research grant N00014-95-1152, National Institute of Mental Health grant 5P01-MH52194, and a grant from the Sankyo Company, LTD.

The publication costs of this article were defrayed in part by payment of page charges. This article must therefore be hereby marked "advertisement" in accordance with 18 USC section 1734 solely to indicate this fact.

\section{References}

Albus, J.S. 1971. A theory of cerebellar function. Math. Biosci. 10: 25-61.

Berthier, N.E. and J.W. Moore. 1986. Cerebellar Purkinje cell activity related to the classically conditioned nictitating membrane response. Exp. Brain Res. 63: 341-350.

1990. Activity of deep cerebellar nuclear cells during classical conditioning of nictitating membrane extension in rabbits. Exp. Brain Res. 83: 44-54.

Blaxton, T.A., T.A. Zeffiro, J.D.E. Gabrieli, S.Y. Bookheimer, M.C. Carrillo, W.H. Theodore, and J.F. Disterhoft. 1996. Functional mapping of human learning-a positron emission tomography activation study of eyeblink conditioning. J. Neurosci. 16: 4032-4040.
Bloedel, J.R. and V. Bracha. 1995. On the cerebellum, cutaneomuscular reflexes, movement control and the elusive engrams of memory. Behav. Brain Res. 68: 1-44.

Bracha, V., M.L. Webster, N.K. Winters, K.B. Irwin, and J.R. Bloedel. 1994. Effects of muscimol inactivation of the cerebellar interposed-dentate nuclear-complex on the performance of the nictitating-membrane response in the rabbit. Exp. Brain Res. 100: 453-468.

Clark, R.E. and D.G. Lavond. 1993. Reversible lesions of the red nucleus during acquisition and retention of a classically conditioned behavior in rabbits. Behav. Neurosci. 107: 264-270.

Clark, R.E., A.A. Zhang, and D.G. Lavond. 1992. Reversible lesions of the cerebellar interpositus nucleus during acquisition and retention of a classically conditioned behavior. Behav. Neurosci. 106: 879-888.

Hardiman, M.J., N. Ramnani, and C.H. Yeo. 1996. Reversible inactivations of the cerebellum with muscimol prevent the acquisition and extinction of conditioned nictitating membrane responses in the rabbit. Exp. Brain Res. 110: $235-247$.

Hikosaka, O. and R.H. Wurtz. 1985a. Modification of saccadic eye movements by GABA-related substances. I. Effect of muscimol and bicuculline in monkey superior colliculus. J. Neurophysiol. 53: 266-291.

1985b. Modification of saccadic eye movements by GABA-related substances. II. Effects of muscimol in monkey substantia nigra pars reticulata. J. Neurophysiol. 53: 292-308.

Krupa, D.J. 1993. Localization of the essential memory trace for a classically conditioned behavior. Ph.D. thesis, University of Southern California, Los Angeles, CA.

Krupa, D.J. and R.F. Thompson. 1995. Inactivation of the superior cerebellar peduncle blocks expression but not acquisition of the rabbit's classically conditioned eye-blink response. Proc. Natl. Acad. Sci. 92: 5097-5101.

Krupa, D.J., J.K. Thompson, and R.F. Thompson. 1993. Localization of a memory trace in the mammalian brain. Science 260: 989-991.

Krupa, D.J., J. Weng, and R.F. Thompson. 1996. Inactivation of brainstem motor nuclei blocks expression but not acquisition of the rabbit's classically conditioned eyeblink response. Behav. Neurosci. 110: 219-227.

Lavond, D.G., T.L. Hembree, and R.F. Thompson. 1985 Effect of kainic acid lesions of the cerebellar interpositus nucleus on eyelid conditioning in the rabbit. Brain Res. 326: 179-182.

Lavond, D.G., B.J. Knowlton, J.E. Steinmetz, and R.F. Thompson. 1987. Classical conditioning of the rabbit eyelid response with a mossy-fiber stimulation CS: II. Lateral reticular nucleus stimulation. Behav. Neurosci. 101: 676-682.

Lavond, D.G., J.J. Kim, and R.F. Thompson. 1993. Mammalian brain substrates of aversive classical conditioning. Annu. Rev. Psychol. 44: 317-342.

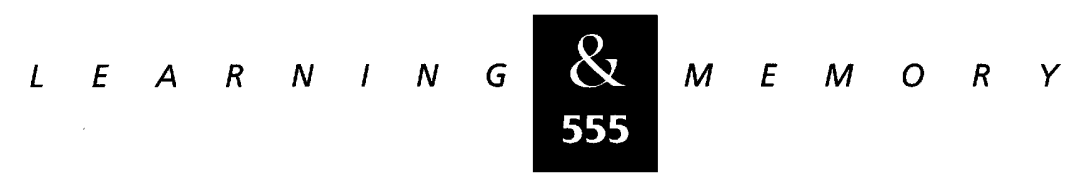


Lincoln, J.S., D.A. McCormick, and R.F. Thompson. 1982. Ipsilateral cerebellar lesions prevent learning of the classically conditioned nictitating membrane/eyelid response. Brain Res. 242: 190-193.

Lisberger, S.G. 1988. The neural basis for learning of simple motor skills. Science 242: 728-735.

Logan, C.G. and S.T. Grafton. 1995. Functional anatomy of human eyeblink conditioning determined with regional cerebral glucose metabolism and positron-emission tomography. Proc. Natl. Acad. Sci. 92: 7500-7504.

Marr, D. 1969. A theory of cerebellar cortex. J. Physiol. 202: $437-470$.

Martin, J.H. 1991. Autoradiographic estimation of the extent of reversible inactivation produced by microinjection of lidocaine and muscimol in the rat. Neurosci. Lett. 127: 160-164.

Martin, J.H. and C. Ghez. 1988. Red nucleus and motor cortex: Parallel motor systems for the initiation and control of skilled movement. Behav. Brain Res. 28: 217-223.

Mauk, M.D., J.E. Steinmetz, and R.F. Thompson. 1986. Classical conditioning using stimulation of the inferior olive as the unconditioned stimulus. Proc. Natl. Acad. Sci. 83: 5349-5353.

McBride, R.L. and W.R. Klemm. 1968. Stereotaxic atlas of the rabbit brain, based on the rapid method of photography of frozen, unstained sections. Commun. Behav. Biol. 2: $179-215$.

McCormick, D.A. and R.F. Thompson, 1984a. Cerebellum: Essential involvement in the classically conditioned eyelid response. Science 223: 296-299.

1984b. Neuronal responses of the rabbit cerebellum during acquisition and performance of a classically conditioned nictitating membrane-eyelid response. J. Neurosci. 4: 2811-2822.

Mink, J.W. and W.T. Thach. 1991. Basal ganglia motor control. III. Pallidal ablation: Normal reaction time, muscle cocontraction, and slow movement. J. Neurophysiol. 65: $330-351$.

Molchan, S.E., T. Sunderland, A.R. McIntosh, P. Herscovitch, and B.G. Schreurs. 1994. A functional anatomical study of associative learning in humans. Proc. Natl. Acad. Sci. 91: 8122-8126.

Nordholm, A.F., J.K. Thompson, C. Dersarkissian, and R.F. Thompson. 1993. Lidocaine infusion in a critical region of cerebellum completely prevents learning of the conditioned eyeblink response. Behav. Neurosci. 107: 882-886.

Perrett, S.P. and M.D. Mauk. 1995. Extinction of conditioned eyelid responses requires the anterior lobe of cerebellar cortex. J. Neurosci. 15: 2074-2080.
Ramirez, O.A., A.F. Nordholm, D. Gellerman, J.K. Thompson, and R.F. Thompson. 1997. The conditioned eyeblink response: A role for the GABA-B receptor. Pharmacol. Biochem. Behav. (in press).

Ramnani, N. and C.H. Yeo. 1996. Reversible inactivations of the cerebellum prevent the extinction of conditioned nictitating membrane responses in rabbits. J. Physiol. 495.1: 159-168.

Steinmetz, J.E., D.J. Rosen, P.F. Chapman, D.G. Lavond, and R.F. Thompson. 1986. Classical conditioning of the rabbit eyelid response with a mossy-fiber stimulation CS: I. Pontine nuclei and middle cerebellar peduncle stimulation. Behav. Neurosci. 100: 878-887.

Steinmetz, J.E., D.G. Lavond, and R.F. Thompson. 1989. Classical conditioning in rabbits using pontine nucleus stimulation as a conditioned stimulus and inferior olive stimulation as an unconditioned stimulus. Synapse 3: 225-233.

Steinmetz, J.E., D.G. Lavond, D. Ivkovich, C.G. Logan, and R.F. Thompson. 1992. Disruption of classical eyelid conditioning after cerebellar lesions: Damage to a memory trace system or a simple performance deficit? J. Neurosci. 12: $4403-4426$.

Thach, W.T., H.P. Goodkin, and J.G. Keating. 1992. The cerebellum and the adaptive coordination of movement. Annu. Rev. Neurosci. 15: 403-442.

Thompson, R.F. and D.J. Krupa. 1994. Organization of memory traces in the mammalian brain. Annu. Rev. Neurosci. 17: 519-549.

Thompson, R.F. and J.J. Kim. 1996. Memory-systems in the brain and localization of a memory. Proc. Natl. Acad. Sci. 93: 13438-13444

Van Neerven, J., O. Pompeiano, and H. Collewijn. 1989. Depression of the vestibulo-ocular and optokinetic responses by intrafloccular microinjection of GABA-A and GABA-B agonists in the rabbit. Arch. Italiennes de Biol. 127: 243-263.

Yeo, C.H. 1991. Cerebellum and classical conditioning of motor responses. Ann. N.Y. Acad. Sci. 627: 292-304.

Yeo, C.H., M.J. Hardiman, and M. Glickstein. 1985. Classical conditioning of the nictitating membrane response of the rabbit. I. Lesions of the cerebellar nuclei. Exp. Brain Res. 60: 87-98.

Received February 18, 1997; accepted in revised form April 19,1997

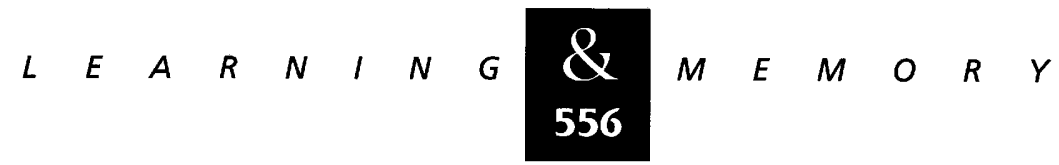




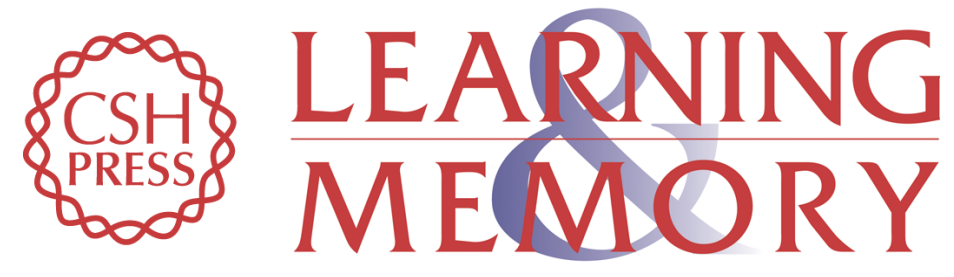

\section{Reversible inactivation of the cerebellar interpositus nucleus completely prevents acquisition of the classically conditioned eye-blink response.}

D J Krupa and R F Thompson

Learn. Mem. 1997, 3:

Access the most recent version at doi:10.1101//m.3.6.545

References This article cites 41 articles, 12 of which can be accessed free at: http://learnmem.cshlp.org/content/3/6/545.full.html\#ref-list-1

License

Email Alerting Receive free email alerts when new articles cite this article - sign up in the box at the Service top right corner of the article or click here. 